\title{
Electrochemical detection and imaging of reactive oxygen species in single living cells
}

Alexander Vaneev ${ }^{1}$, Roman Timoshenko ${ }^{2}$, Vasilii Kolmogorov ${ }^{3}$, Helena Lopatukhina ${ }^{4}$, Peter Gorelkin ${ }^{4}$, Alexander Erofeev ${ }^{4}$, Natalia Klyachko ${ }^{5}$, Yuri Korchev ${ }^{6}$, Alexander Majouga ${ }^{7}$ and Pavel Novak ${ }^{8}$

${ }^{1}$ National University of Science and Technology «MISiS», Russia, ${ }^{2}$ National University of Science and Technology "MISIS", Moscow, Moskva, Russia, ${ }^{3}$ National University of Science and Technology «MISiS», Moscow, Russia, Moskva, Russia, ${ }^{4}$ National University of Science and Technology «MISiS», Moscow, Russia, Russia, ${ }^{5}$ Lomonosov Moscow State University, Moscow, Russia, Russia, ${ }^{6}$ National University of Science and Technology "MISIS", Moscow, Russia, ${ }^{7}$ D. Mendeleev University of Chemical Technology, Russia, ${ }^{8}$ National University of Science and Technology «MISiS», Moscow, Russia, United States

Oxidative stress is an area of significant interest, as it is an important marker for carcinogenic cell behavior, in cell signaling and inflammatory response. The study of reactive oxygen species (ROS) levels may represent one possibility to research the effects of drugs in cancer cells. ROS are associated with induction of apoptosis and are released from cells during apoptosis, play a crucial role in the development of cancer and neurodegenerative diseases. Nowadays, there is the problem of methods for treating cancer tumors, and quickly evaluation of the anticancer drugs efficiency is the priority. The ROS determination using nanosensors in single cells has gained increasing attention. However, traditional fluorescent dyes have a number of disadvantages. Here, we have developed an electrochemical method for ROS detection in single living cells and imaging. Using this method, it is possible to evaluate the effect of the new developed drugs on the cells. We have previously reported the fabrication of disk-shaped carbon nanoelectrodes based on a quartz nanopipete that were functionalized by platinum for improvement of their electroactive properties. ROS measurements within melanoma cells were carried out with minimal disruption of cell function (Actis et al., 2014). Recently, a new type of nanoelectrode based on a quartz nanopipete with better platinum adhesion was made and we have developed a new method for an in vitro study of the toxicity of magnetic nanoparticles based on the measurement of intracellular ROS (Erofeev et al., 2018). We have also measured ROS levels under riboflavin blue light irradiation in single melanoma cells using an electrochemical nanoprobe in real-time (Akasov et al., 2019). We demonstrate a new method for determining the ROS response to chemotherapeutics within cancer single cells (Vaneev et al., 2020). Thus, exploiting small nanoelectrodes for the life sciences can bring about a deeper understanding of physiological processes occurring inside living cells noninvasively. Also we tested the method for mapping the sample surface with simultaneous obtaining of the ROS ( $\mathrm{H} 2 \mathrm{O} 2)$ image using fabricated two- 
channel nanosensors. One channel was filled by buffer solution for obtaining topography, second channel was filled by pyrolytic carbon with following Pt modification. For ROS imaging, a nanocapillary was filled with hydrogen peroxide and located parallel to the bottom of a Petri dish filled with a buffer solution, then the nanocapillary was scanned by two-channel nanosensor. Scanning was performed using an unmodified channel filled with buffer solution at $+200 \mathrm{mV}$ vs. $\mathrm{Ag} / \mathrm{AgCl}$. The ROS was measured simultaneously with scanning the nanocapillary surface at a potential of $+800 \mathrm{mV}$ vs. Ag / $\mathrm{AgCl}$. Thus, using these sensors, it is possible to determine and image intracellular and extracellular ROS in single living cells. The nanoelectrode measurements were supported by Russian Science Foundation (grant № 19-79-30062). The biological experiments with cell cultures were supported by the 'NUST MISiS' grant no. K4-2017-048.

\section{References}

Actis, P., Tokar, S., Clausmeyer, J., Babakinejad, B., Mikhaleva, S., Cornut, R., Takahashi, Y., López Córdoba, A., Novak, P., Shevchuck, A. I., López Córdoba, A., Novak, P., Shevchuck, A. I., Dougan, J. A., Kazarian, S. G., Gorelkin, P. V., Erofeev, A. S., Yaminsky, I. V., Unwin, P. R., Schuhmann, W., Klenerman, D., Rusakov, D. A., Sviderskaya, E. V. \& Korchev, Y. E. (2014). Electrochemical nanoprobes for single-cell analysis. Acs Nano 8, 875-884.

Akasov, R. A., Sholina, N. V., Khochenkov, D. A., Alova, A. V., Gorelkin, P. V., Erofeev, A. S., Generalova, A. N. \& Khaydukov, E. V. (2019). Photodynamic therapy of melanoma by blue-light photoactivation of flavin mononucleotide. Scientific Reports 9, 9679.

Erofeev, A., Gorelkin, P., Garanina, A., Alova, A., Efremova, M., Vorobyeva, N., Edwards, C., KorcheV, Y. \& MAJOugA, A. (2018). Novel method for rapid toxicity screening of magnetic nanoparticles. Scientific reports 8, 7462.

Vaneev, A. N., Gorelkin, P. V., Garanina, A. S., Lopatukhina, H. V., Vodopyanov, S. S., Alova, A. V., Ryabaya, O. O., Akasov, R. A., Zhang, Y., Novak, P., Salikhov, S. V., Abakumov, M. A., Takahashi, Y., Edwards, C. R. W., Klyachko, N. L., Majouga, A. G., KorcheV, Y. E. \& Erofeev, A. S. (2020). In Vitro and In Vivo Electrochemical Measurement of Reactive Oxygen Species After Treatment with Anticancer Drugs. Analytical Chemistry 92, 8010-8014. 